\section{EXPERIÊNCIA COM A MÚSICA, BEM- ESTAR E SAÚDE MENTAL:}

QUE RELAÇÕES?

revistamúsica | Vol. 18, n.2 |

pp. 30-45 | dez. 2018

\section{AleXANDRA MARQUeS \\ Universidade Fernando Pessoa - Porto, Portugal|26073@ufp.edu.pt}

\section{CARLa Fonte}

Universidade Fernando Pessoa - Porto, Portugal | cfonte@ufp.edu.pt

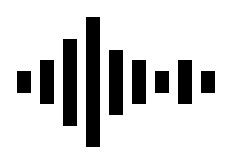

\section{EXPERIENCE WITH MUSIC, WELLNESS AND MENTAL}

HEALTH:

\section{WHAT RELATIONS?}

\section{RESUMO}

A literatura tem sublinhado a importância de se estudar a relação entre a música e a saúde mental. Neste sentido apresenta-se um estudo cujo objetivo é caraterizar como os indivíduos experienciam a música e sua relação com o bem-estar e saúde mental. A amostra é constituída por 255 adultos, com idades compreendidas entre os 18 e 67 anos. Foram utilizados para a recolha de dados o Questionário da Experiência com Música, Escala Continuum de Saúde Mental e a Escala de Ansiedade, Depressão e Stress. Os resultados indicam associações entre experiência com a música não só com relação aos níveis de bemestar, mas também relacionando-se com os níveis de ansiedade, stress e depressão. As diferenças se dão de acordo com a relação que os participantes têm com a música. Estes resultados sugerem que a música pode ter um impacto positivo ou negativo na saúde mental e bem-estar dos indivíduos.

\section{PALAVRAS-CHAVE}

Música. Saúde mental. Bem-estar.

\section{ABSTRACT}

The literature has emphasized the importance of studying the relation between music and mental health. In this sense a study is presented that aim to characterize how individuals experience music and its relation to well-being and mental health. The sample consists of 255 adults, aged between 18 and 67 years. The Music Experience Questionnaire, Continuum of Mental Health Scale and the Anxiety, Depression and Stress Scale were used for data collection. The results indicate significant associations between some dimensions of the experience with music, wellbeing levels, but also with anxiety, stress and depression levels, and differences according to the relationship that the participants have with music. These results suggest that music can have a positive or negative impact on the mental health and well-being of individuals.

\section{KEYWORDS}

Music. Mental health. Wellbeing. 


\title{
Experiência com a música, bem-estar e saúde mental: que relações? ${ }^{1}$
}

\author{
ALEXANDRA MARQUES \\ Universidade Fernando Pessoa, Porto | 26073@ufp.edu.pt
}

CARLA FONTE

Universidade Fernando Pessoa, Porto | cfonte@ufp.edu.pt

\section{Introdução}

De acordo com Levitin (2013), temos tendência para aceitar e mesmo para desfrutar inteiramente o poder que a música tem de nos fazer experienciar diferentes sentimentos. Para além disso, segundo SACKS (2008), independentemente de a procurarmos ou não, ou de nos vermos ou não como pessoas particularmente «musicais», a música, para a maioria dos indivíduos, possui uma tremenda força, sendo um dos mais poderosos meios de induzir emoções, significa que a sua capacidade de exercer influência no nosso bem-estar é forte, intemporal, independentemente da cultura (RICKARD, 2014).

O reconhecimento, seja pela comunidade científica ou pela população em geral, que a música nos pode influenciar profundamente e de diversas formas, tem implícito que esta poderá ter um efeito positivo ou negativo na vida em geral dos indivíduos (VÄSTFJäLL et al., 2012). O campo da psicologia da música é amplo e abrange essencialmente tudo o que se refere à experiência e comportamento humano relacionados com a música (GABRIELSSON, 2011). A música dirige-se às duas partes da natureza humana - tanto emocional como intelectual (SACKS, 2008). Do campo da neurociência cognitiva surgem evidências biológicas da influência direta da música na emoção humana (JUSLIN; SLOBODA, 2012) ainda que muitas questões sobre a base neurobiológica das emoções musicais permaneçam por responder. Há de facto evidências de que as emoções musicais dependem de um caminho emocional especializado que convoca várias estruturas subcorticais e corticais que podem ser partilhados, pelo menos em parte, com outros sistemas biologicamente importantes (PERETZ, 2012). Do estudo das funções psicológicas da música no dia a dia emergem três dimensões: cognitiva, emocional e social (NORTH; HARGREAVES, 1999). A experiência da música é determinada na interação de fatores presentes na música, no indivíduo e na situação (GABRIELSSON, 2011). Existem assim estudos que visam os aspetos particulares da música (tom, tempo, ritmo, etc.), estudos compreensivos de como a música é criada, interpretada e experienciada, e estudos de como usamos a música e a importância que ela tem na vida de indivíduos. Estes estudos referem que a música é uma atividade de lazer com a capacidade de melhorar o bem-estar (RICKARD; MCFERRAN, 2012 apud MACDONALD et al., 2012; CHIN; RICKARD, 2014) e ter impacto sobre os sintomas de doença mental, como

\footnotetext{
${ }^{1}$ Nota do Editor: neste artigo foi mantida a ortografia do idioma português de Portugal.
} 
depressão e ansiedade (MARATOS et al., 2008; BRADT et al., 2011; SIEDLIECKI; GOOD, 2006 apud CHIN; RICKARD, 2014).

Como atingir o bem-estar sempre foi uma questão fundamental da vida humana (HENDERSON; KNIGHT, 2012) e na literatura encontram-se descritas duas perspetivas distintas acerca do bem-estar, sendo que uma se centra no bem-estar hedônico (ou bem-estar emocional e/ou subjetivo) e outra no bem-estar eudaimônico (bem-estar psicológico) (RYAN; DECI, 2001; HENDERSON; KNIGHT, 2012). Segundo estas perspetivas, os componentes de bem-estar subjetivo (BES) são um conjunto maior de emoções positivas do que de emoções negativas e um sentimento de satisfação com a vida (DIENER et al., 1999 apud GROARKE; HOGAN, 2015), enquanto que na abordagem eudaimônica a definição de bem-estar engloba a dimensão do propósito e significado de vida (SELIGMAN, 2011).

A relação entre a música, saúde e bem-estar é complexa e envolve inúmeros aspetos e desafios (MACDONALD et al., 2012). A literatura tem salientado a importância de estudar a relação entre a experiência com música e as dimensões do bem-estar (bem-estar emocional, social e psicológico). Neste âmbito desenvolveu-se um estudo que tem como objetivo analisar como os indivíduos experienciam a música no seu dia-a-dia e que relações se podem estabelecer entre as dimensões da experiência com a música com o bem-estar e sintomas de psicopatologia, tais como depressão, ansiedade e stress. Pretende-se assim contribuir para um melhor entendimento de como a música pode influenciar a saúde mental das pessoas.

\section{Método}

O presente estudo é quantitativo, transversal, descritivo-correlacional recorrendo a instrumentos psicométricos de recolha de dados.

\section{Participantes}

A recolha da amostra foi feita através de meio digital sendo constituída por 255 participantes, 79 (31.0\%) do sexo masculino e 176 (69.0\%) do sexo feminino. A idade média dos participantes é de 35,36 (Desvio Padrão = 12.50), variando entre 18 e 67 anos. No que se refere ao estado civil, a maioria dos participantes é solteiro ( $n=130 ; 51.0 \%)$, seguindo-se os indivíduos que se encontram casados ( $n=85 ; 33.3 \%)$. Quanto às habilitações acadêmicas, $40 \%$ da amostra possui o ensino superior, seguindo-se aqueles que apresentam pós-graduação (31.4\%) e ensino secundário (23.9\%). No tocante à situação profissional, a maioria dos participantes é assalariado (47.1\%), seguindo-se os estudantes (26.7\%). No que concerne à experiência musical, quanto ao tipo de relação com a música, a maioria dos participantes apresenta-se como ouvinte ( $n=215$; 84.3\%), os restantes se apresentam tendo outro tipo de relação com a música, seja performer, compositor ou professor, $(n=40 ; 15.7 \%)$. Do total dos participantes $31.3 \%$ ( $n=135)$ afirmam terem sido os pais a introduzi-los à música e 30.3\% $(n=131)$ reportam não ter existido ninguém a introduzi-los à música. Quanto a contextos nos quais os participantes ouvem música 39,0\% ( $n$ = 221) responde ouvir música em background (isto é em casa, no local de trabalho), 33,9\% ( $n=$ 
192) que ouve música enquanto se desloca e 20,6\% $(n=117)$ ouve música com objetivo principal (isto é em concertos, locais recreativos noturnos). Da amostra 72,5\% dos participantes não tocam qualquer tipo de instrumentos $(n=185)$. Dos participantes que responderam afirmativamente 40,0\%, $(n=36)$ tocam instrumento de corda (e.g., viola, violino, etc.) e 35,6\% ( $n=32)$ instrumento de percussão. No que concerne ao estilo de música os três estilos que os participantes mais ouvem/compõem/interpretam são a música Pop (14,6\%, $n=147)$, o Rock'n'Roll (12,4\%, $n=$ 124) e música Clássica $(8.2 \%, n=82)$.

\section{Instrumentos}

Tendo presente os objetivos da investigação, recorreu-se aos seguintes instrumentos como forma de recolha dos dados pretendidos: o Questionário da Experiência com Música - Versão Curta (QBEM), versão portuguesa do Brief MEQ, a Escala de Ansiedade, Depressão e Stress (EADS-21), e a Escala Continuum de Saúde Mental (MHC-SF).

\section{Questionário Breve da Experiência da Música (QBEM) - Versão portuguesa do Brief Music Experience Questionnaire (Brief MEQ)(MARQUES, 2017; MARQUES; FONTE, 2017).}

A versão portuguesa do Brief Music Experience Questionnaire (Brief MEQ) (WERNER et al., 2006), é composto por 53 itens, as respostas são dadas numa escala tipo Likert de 5 pontos. Estes 53 itens constituem seis escalas. Os itens abrangem uma vasta gama de temas relativos ao lugar da música na vida de uma pessoa, independentemente do estilo de música, e representam conteúdo relevante para não-músicos, bem como para músicos. As escalas são: (1) compromisso com a música, que se refere à centralidade da procura de experiências musicais na vida da pessoa (2) aptidão musical inovadora, decorrente de autorrelatos da capacidade de desempenho musical, bem como a capacidade de gerar obras e temas musicais (3) conexão social, que avalia a experiência de através da música de ser movido e animado de forma orientada para o grupo; (4) reação afetiva, que indaga sobre reações afetivas e espirituais à música; (5) efeito psicoativo positivo, que se refere ao efeito calmante, energizante e reações integradoras e, (6) reação comportamental à música que avalia reações predominantemente motoras incluindo cantarolar e balancear ao som da música (WERNER et al., 2006; 2009).

\section{Escala Continuum de Saúde Mental (MHC-SF) - versão portuguesa}

Esta escala avalia as três dimensões da saúde mental positiva - bem-estar emocional, psicológico e social, sendo um instrumento composto por 14 itens em que cada item representa o sentimento de bem-estar, bem como a frequência com que o experienciou, classificada numa escala tipo Likert de 6 pontos (LAMERS et al., 2011). Os itens encontram-se distribuídos pelas três dimensões: o bem-estar emocional composto por 3 itens, o bem-estar social por 5 itens e o bem-estar psicológico por 6 itens, categorizando os indivíduos em três categorias - flourishing, languishing e saúde mental moderada. O estado de flourishing consiste numa combinação de bem-estar emocional e funcionamento positivo na vida, que se reflete no bemestar psicológico e no bem-estar social (KEYES, 2002; 2005; WESTERHOF; KEYES, 2010). Por sua vez o estado de languishing, é definido como um estado de saúde 
mental no qual o indivíduo apresenta baixos níveis de bem-estar, embora não se encontre deprimido (KEYES, 2002; 2005; WESTERHOF; KEYES, 2010). Já a saúde mental moderada verifica-se quando nem os critérios de flourishing nem de languishing são preenchidos (KEYES, 2002; WESTERHOF; KEYES, 2010).

\section{Escala de Ansiedade, Depressão e Stress (EADS)}

Desenvolvida por Pais-Ribeiro, Honrado e Leal em 2004, tem como objetivo avaliar a ansiedade, a depressão e o Stress, compondo desta forma as suas três subescalas na qual cada uma delas é avaliada através de sete itens, formando um total de 21 (PAIS-RIBEIRO et al., 2004; NORTON, 2007). Cada dimensão inclui itens que pretendem avaliar aspetos teoricamente inclusivos nessas mesmas dimensões. Os indivíduos devem responder de acordo com a frequência ou gravidade, numa escala tipo Likert de 4 pontos, tendo em conta a última semana (PAISRIBEIRO et al., 2004; NORTON, 2007). A subescala referente à ansiedade envolve conceitos como ativação do sistema nervoso autónomo, efeitos músculo esqueléticos, ansiedade situacional e experiências subjetivas de ansiedade. A subescala da depressão inclui disforia, desânimo, desvalorização da vida, auto-depreciação, falta de interesse ou de envolvimento, anedonia e inércia. Por fim, a subescala do stress abrange dificuldade em relaxar, excitação nervosa, agitação, irritabilidade ou reação exagerada e impaciência (PAIS-RIBEIRO et al., 2004).

\section{Procedimento}

Para a realização do presente estudo procedeu-se primeiramente, ao pedido de autorização aos autores dos instrumentos utilizados. Desta forma obteve-se através do Professor Doutor Paul Werner a autorização para a tradução e uso do BMEQ. No que respeita à Escala Continuum de Saúde Mental - versão reduzida (MHC-SF), a Professora Doutora Carla Fonte autorizou a sua utilização. Quanto à Escala de Ansiedade, Depressão e Stress (EADS), esta encontra-se publicada, não sendo necessária a autorização por parte dos seus autores. Posteriormente, o projeto deste estudo foi submetido à Comissão de Ética da Universidade Fernando Pessoa, que declarou um parecer favorável ao desenvolvimento do mesmo. Após a obtenção do deferimento da Comissão de Ética da Universidade de afiliação dos autores do estudo, todos questionários foram transpostos para suporte online de forma a facilitar a participação dos sujeitos e a obter um maior número de dados.

De forma a resguardar e informar os participantes de todos os esclarecimentos quanto aos instrumentos e ao estudo em si, salvaguardando o anonimato e a confidencialidade, foi numa primeira fase apresentada aos participantes declaração de assentimento da participação no estudo, sendo este um fator necessário para responder ao questionário sociodemográfico e aos restantes instrumentos.

A recolha de dados, foi feita por meio informático, de forma não-aleatória e pelo método Snowball, através da divulgação do link do estudo num grupo inicial, sendo pedido a esses mesmos participantes a divulgação do link do estudo a outros membros da população por forma a aumentar o número de participantes. Todos os instrumentos estiveram disponíveis durante o 
tempo de resposta num link especificamente criado através do Google Docs de forma a que os sujeitos tivessem acesso ao mesmo. Sendo que, ao serem reunidas as respostas necessárias o link tornou-se indisponível. As respostas dadas pelos sujeitos foram sumariadas num documento Excel criado automaticamente pelo Goggle Docs e posteriormente procedeu-se a análise estatística dos dados recolhidos recorrendo ao programa Statistical Package for the Social Sciences-SPSS (versão 22). Foram utilizadas análises descritivas, o Teste de Associação Coeficiente de Correlação de Spearman e Testes de Diferenças em Contexto de Design InterSujeitos, nomeadamente o Teste de Mann-Whitney (Mann-Whitney Test, $U$ ) para Amostras Independentes.

\section{Resultados}

Iniciou-se a análise estatística por determinar a normalidade da distribuição das variáveis intervalares e homogeneidade das variâncias. A análise exploratória de dados para a amostra ( $N$ =255) revelou não estarem cumpridos os pressupostos subjacentes à utilização de testes paramétricos, pelo que os testes utilizados são não paramétricos (ALMEIDA; FREIRE, 2008).

Em seguida apresentam-se os resultados de acordo com os objetivos, e organizados, através dos pontos que se seguem.

\section{Caraterização da amostra relativamente à experiência com a música}

No que diz respeito ao BMEQ é possível verificar-se que, atendendo às pontuações mínimas e máximas possíveis, os participantes pontuam relativamente alto nas subescalas conexão social, reação emocional, efeito psicoativo positivo e reação comportamental à música. A subescalas compromisso com a música e aptidão musical inovadora são as que se verificam menor pontuação (Tabela 1).

\begin{tabular}{|c|c|c|c|c|}
\hline \multicolumn{5}{|c|}{$\begin{array}{l}\text { Tabela } 1 \\
\text { Apresentação dos Resultados Relativos } \\
\text { Medidas Descritivas } \\
\end{array}$} \\
\hline & \multicolumn{4}{|c|}{ Amostra $(N=\mathbf{2 5 5})$} \\
\hline & MÍNIMO & MÁXIMO & MÉDIA & $\begin{array}{l}\text { DESVIO } \\
\text { PADRÃO }\end{array}$ \\
\hline Compromisso com a Música & 1.00 & 5,00 & 2,70 & 0,88 \\
\hline Aptidão Musical Inovadora & 1.00 & 5,00 & 2,02 & 1,02 \\
\hline Conexão Social & 1.00 & 5,00 & 3,30 & 0,87 \\
\hline Reação Afetiva & 1.00 & 5,00 & 4,42 & 0,40 \\
\hline Efeito Psicoativo Positivo & 1.00 & $5, \mathrm{OO}$ & 3,75 & 0,85 \\
\hline Reação Comportamental à Música & 1.00 & 5,00 & 3,91 & 0,75 \\
\hline
\end{tabular}




\section{Caraterização da saúde mental dos participantes relativamente aos níveis de bem-estar e de psicopatologia}

Com relação à escala de bem-estar é possível aferir que os participantes pontuam relativamente alto (Tabela 2), concretamente para as subescalas de BEE (13,77) e BEP (28.04). É no BESo $(18,52)$ que as pontuações se revelam mais baixas.

\begin{tabular}{lcccc}
\hline $\begin{array}{l}\text { Tabela } 2 \\
\text { Apresentação dos Resultados Relativos a Bem-estar Emocional, Bem-estar Social, Bem-estar } \\
\text { Psicológico utilizando Médias }\end{array}$ \\
\cline { 2 - 4 } & \multicolumn{4}{c}{ Desvios-padrão como Medidas Descritivas } \\
\cline { 2 - 4 } & MíNIMO & MÁXIMO & MÉDIA & DESVIO PADRÃO \\
\hline Bem-estar Emocional & 300 & 18.00 & 13.77 & 2.95 \\
Bem-estar Social & 5.00 & 30.00 & 18.52 & 4.92 \\
Bem-estar Psicológico & 6.00 & 36.00 & 28.04 & 5.04 \\
\hline
\end{tabular}

No que diz respeito aos níveis de depressão, ansiedade e stress, é possível verificar que os participantes pontuam relativamente baixo nestes quesitos, apresentando assim baixos níveis de psicopatologia (Tabela 3).

\begin{tabular}{|c|c|c|c|c|}
\hline \multirow{2}{*}{\multicolumn{5}{|c|}{$\begin{array}{l}\text { Tabela } 3 \\
\text { Apresentação dos Resultados Relativos à Escala de Ansiedade, Depressão e Stress (EADS-21) o } \\
\text { utilizando Médias e Desvios-padrão como Medidas Descritivas } \\
\text { Amostra }(\boldsymbol{N}=\mathbf{2 5 5})\end{array}$}} \\
\hline & & & & \\
\hline \multirow[b]{2}{*}{ Depressão } & MÍNIMO & MÁXIMO & MÉDIA & DESVIO PADRÃO \\
\hline & 0.00 & 21.00 & 4.28 & 4.65 \\
\hline Ansiedade & 0.00 & 21.00 & 3.62 & 4.00 \\
\hline Stress & 0.00 & 21.00 & 7.62 & 5.14 \\
\hline
\end{tabular}

Atendendo às pontuações mínimas e máximas possíveis nas escalas supramencionadas é possível verificar que os participantes percecionam níveis relativamente elevados de bem-estar e baixos níveis de psicopatologia (depressão, ansiedade e stress).

\section{Relação entre as dimensões da experiência com música e os níveis de bem-estar e psicopatologia}

No que concerne às relações entre as dimensões da experiência da música e os níveis de bem-estar verifica-se a existência de uma associação positiva entre dimensão do efeito psicoativo positivo e o nível de bem-estar psicológico $\left(r_{s}=.143 ; p<, 01\right)$, ou seja, maior nível de efeito psicoativo positivo está associado a maiores níveis de saúde mental e bem-estar psicológico. Também a dimensão reação comportamental à música de associa positivamente aos níveis de bem-estar psicológico $\left(r_{\mathrm{s}}=.139 ; p<, 01\right)$, indicando que quanto maior o nível reação comportamental à música maiores os níveis de saúde mental e bem-estar psicológico.

No que diz respeito à relação entre as dimensões da experiência da música e os níveis de psicopatologia, constata-se uma correlação positiva entre o grau de aptidão musical inovadora e o nível de ansiedade $\left(r_{\mathrm{s}}=.166 ; p<, 01\right)$, indicando que quanto maior nível de aptidão musical inovadora maior o nível de ansiedade. Verificou-se também uma associação positiva entre o nível 
de conexão social e o nível de depressão $\left(r_{\mathrm{s}}=.157 ; p<\right.$,o1 $)$ e o nível de ansiedade $\left(r_{\mathrm{s}}=.199 ; p<\right.$ ,01), sugerindo que quanto maior o grau de conexão social maiores também níveis de depressão e ansiedade na amostra. Constata-se também uma correlação positiva entre o efeito psicoativo positivo e o nível de ansiedade $\left(r_{s}=.180 ; p<, 01\right)$ e o nível de stress $\left(r_{s}=.145 ; p<, 01\right)$. Assim, maior nível de efeito psicoativo positivo está associado maiores níveis ansiedade e stress.

\begin{tabular}{|c|c|c|c|c|c|c|}
\hline \multicolumn{7}{|c|}{$\begin{array}{l}\text { Tabela } \mathbf{4} \\
\text { Correlações entre a Experiência da Música (BMEQ) e o bem-estar emocional, o bem-estar social, bem-estar } \\
\text { psicológico, a depressão, a ansiedade e o stress. }\end{array}$} \\
\hline & \multicolumn{6}{|c|}{$N=255$} \\
\hline & $\begin{array}{l}\text { COMPROMISSO } \\
\text { COM A MÚSICA }\end{array}$ & $\begin{array}{l}\text { APTIDÃO } \\
\text { MUSICAL } \\
\text { INOVADORA }\end{array}$ & $\begin{array}{c}\text { CONEXÃO } \\
\text { SOCIAL }\end{array}$ & $\begin{array}{l}\text { REAÇÃO } \\
\text { AFETIVA }\end{array}$ & $\begin{array}{c}\text { EFEITO } \\
\text { PSICOATIVO } \\
\text { POSITIVO }\end{array}$ & $\begin{array}{c}\text { REAÇÃO } \\
\text { COMPORTAMENTAL À } \\
\text { MÚSICA }\end{array}$ \\
\hline & $r_{s}$ & $r_{s}$ & $r_{s}$ & $r_{s}$ & $r_{s}$ & $r_{s}$ \\
\hline $\begin{array}{l}\text { Bem-estar } \\
\text { Emocional }\end{array}$ & -,019 & ,003 & -,o32 &,- 021 & ,011 & ,013 \\
\hline Bem-estar Social & ,036 & ,021 & ,072 &,- 090 & ,022 & ,o30 \\
\hline $\begin{array}{l}\text { Bem-estar } \\
\text { Psicológico }\end{array}$ & ,049 & ,041 & ,029 & , 043 &, $143^{*}$ &, $139^{*}$ \\
\hline Depressão & ,048 &,- 004 &, $\mathbf{1 5} 7^{*}$ & , 053 & ,096 &,- 034 \\
\hline Ansiedade & 122 &, $166^{* *}$ &, $199^{* * *}$ & , 078 &, $\mathbf{1 8 0}^{* *}$ & ,069 \\
\hline Stress & ,044 & ,082 & 107 & ,065 &, $\mathbf{1 4 5}^{*}$ & 109 \\
\hline
\end{tabular}

Nota: **. A correlação é significativa no nível p< 0,01 (2 extremidades).

*. A correlação é significativa no nível p o,05 (2 extremidades).

\section{Diferenças entre as dimensões da experiência com a música, os níveis bem-estar e psicopatologia}

Os participantes que têm outra relação com a música revelam pontuações mais elevadas em todas as subescalas do BMEQ face às pontuações daquele que são apenas ouvintes (Tabela 5). É nas dimensões compromisso com a música $(U=1159.00 ; p<.001)$, aptidão musical inovadora $(U=572.00, p<.001)$ e efeito psicoativo positivo $U=2080,50, p<$,oo1 que se verificam diferenças significativas. 


\begin{tabular}{|c|c|c|c|c|c|}
\hline $\begin{array}{l}\text { Tabela } 5 \\
\text { Apresentação dos Resultados Relat } \\
\text { como Medidas Descritivas (tipo de }\end{array}$ & $\begin{array}{l}\text { Teste } \\
\text { ocom }\end{array}$ & $\begin{array}{l}\text { Iann-1 } \\
\text { sica) }\end{array}$ & zey ut & do $M \epsilon$ & Desvios-padrão \\
\hline & & & $\begin{array}{r}\text { COM OUT } \\
(n\end{array}$ & $\begin{array}{l}\text { RELAÇÃO } \\
\text { o) }\end{array}$ & \\
\hline & Média & $(D P)$ & Média & $(D P)$ & $U$ \\
\hline BMEQ & & & & & \\
\hline Compromisso com a Música & 2,51 & $(, 78)$ & 3,69 & $(, 69)$ & $1159,00^{* * *}$ \\
\hline Aptidão Musical Inovadora & 1,73 & $(, 74)$ & 3,60 & $(, 90)$ & $572,00^{* * *}$ \\
\hline Conexão Social & 2,65 & $(, 84)$ & 2,81 & $(, 96)$ & $3727,00^{* * *}$ \\
\hline Reação Emocional & 4,40 & $(, 41)$ & 4,53 & $(, 33)$ & $3442,00^{* * *}$ \\
\hline Efeito Psicoativo Positivo & 3,65 & $(, 83)$ & 4,33 & $(, 73)$ & $2080,5^{* * *}$ \\
\hline Reação Comportamental à Música & 3,85 & $(, 75)$ & 4,21 & $(, 65)$ & $3124,50^{* * *}$ \\
\hline Bem-estar Emocional & 13,75 & $(2,84)$ & 13,88 & $(3,56)$ & $3973,50^{* * *}$ \\
\hline Bem-estar Social & 18,50 & $(4,84)$ & 18,65 & $(5,40)$ & $4268,00^{* * *}$ \\
\hline Bem-estar Psicológico & 28,58 & $(5,00)$ & 28,40 & $(5,10)$ & $4102,50^{* * *}$ \\
\hline Depressão & 4,30 & $(4,44)$ & 4,17 & $(5,71)$ & $6587,00^{* * *}$ \\
\hline Ansiedade & 3,47 & $(3,93)$ & 4,43 & $(4,32)$ & $6494,50^{* * *}$ \\
\hline Stress & 7,67 & $(5,11)$ & 7,38 & $(5,37)$ & $6340,50^{* * *}$ \\
\hline
\end{tabular}

Como se pode verificar na Tabela 6, os participantes que tocam algum instrumento musical revelam pontuações mais elevadas em todas as subescalas do BMEQ face às pontuações daquele que não tocam instrumento musical. É nas dimensões compromisso com a música $U=3044,50, p<$,001, aptidão musical inovadora $U=1670,00, p<$,001 e efeito psicoativo positivo $U=4336,00, p<, 001$ que se verificam diferenças significativas.

Não há diferenças significativas entre os participantes que tocam algum instrumento musical ao nível das restantes subescalas do BMEQ. O mesmo se verifica ao nível do bem-estar mental e da psicopatologia. 


\begin{tabular}{|c|c|c|c|c|c|}
\hline \multirow{6}{*}{$\begin{array}{l}\text { Tabela } 6 \\
\text { Apresentação dos Resultados Relati } \\
\text { como Medidas Descritivas (Toca ins }\end{array}$} & $\begin{array}{l}\text { este de } \\
\text { o mus }\end{array}$ & an-Wr & ut & $o M$ & Desvios-padrão \\
\hline & \multicolumn{5}{|c|}{ NÃO TOCA } \\
\hline & \multirow{3}{*}{\multicolumn{2}{|c|}{$\begin{array}{c}\text { TOCA INSTRUMENTO } \\
\text { MUSICAL } \\
(n=70)\end{array}$}} & \multirow{3}{*}{\multicolumn{2}{|c|}{$\begin{array}{l}\text { INSTRUMENTO } \\
\text { MUSICAL } \\
(n=185)\end{array}$}} & \multirow[b]{4}{*}{$U$} \\
\hline & & & & & \\
\hline & & & & & \\
\hline & Média & $(D P)$ & Média & $(D P)$ & \\
\hline Compromisso com a Música & 3,30 & $(, 88)$ & 2,47 & $(, 77)$ & $3044,50 * * *$ \\
\hline Aptidão Musical Inovadora & 3,03 & $(1,05)$ & 1,64 & $(, 70)$ & $1670,00^{* * *}$ \\
\hline Conexão Social & 3,35 & $(, 90)$ & 3,29 & $(, 86)$ & $6117,00^{* * *}$ \\
\hline Reação Emocional & 4,47 & $(40)$ & 4,40 & $(, 40)$ & $5686,50^{* * *}$ \\
\hline Efeito Psicoativo Positivo & 4,06 & $(, 88)$ & 3,64 & $(, 81)$ & $4336,00^{* * *}$ \\
\hline Reação Comportamental à Música & 4,08 & $(, 68)$ & 3,84 & $(, 76)$ & $5252,00^{* * *}$ \\
\hline Bem-estar emocional & 13,54 & $(3,02)$ & 13,86 & $(2,92)$ & $6044,00^{* * *}$ \\
\hline Bem-estar social & 17,73 & $(5,05)$ & 18,82 & $(4,86)$ & $5520,00^{* * *}$ \\
\hline Bem-estar psicológico & 28,07 & $(5,01)$ & 28,52 & $(5,13)$ & $6090,00^{* * *}$ \\
\hline Depressão & 4,36 & $(5,11)$ & 4,25 & $(4,48)$ & $6242,00^{* * *}$ \\
\hline Ansiedade & 4,06 & $(4,53)$ & 3,46 & $(3,78)$ & $6014,50^{* * *}$ \\
\hline Stress & 7,59 & $(5,22)$ & 7,64 & $(5,13)$ & $6368,50^{* * *}$ \\
\hline
\end{tabular}

*** $\mathrm{p}<.001$

Fazendo uma análise mais em detalhe de acordo com o tipo de instrumento que tocam verifica-se que aqueles participantes que tocam um instrumento de cordas, no que concerne à aptidão musical inovadora $(M=2.92 ; D P=.99)$ reportam níveis mais elevados nesta dimensão, face aqueles que não o fazem $(M=1.96 ; D P=.86)$, sendo estas diferenças estatisticamente significativas $(U=978.00, p<, 001)$.

Constatou-se também que há diferenças significativas entre os participantes que tocam instrumento de sopro e aqueles que não o fazem ao nível do compromisso com a música, ( $U$ $=3044.50, p<, 001)$. Os participantes que tocam instrumento de sopro $(M=3.30 ; D P=.88)$ reportam/percecionam níveis mais elevados nesta dimensão face aqueles que não o fazem $(M=$ 2.47; $D P=$.77). Os resultados apontam diferenças significativas na dimensão aptidão musical inovadora $(U=1670.00, p<, 001)$. Os participantes que tocam instrumento de sopro $(M=3.03$; $D P=1.05)$ reportam/percecionam níveis mais elevados de aptidão musical inovadora face aqueles que não o fazem $(M=1.64 ; D P=.70)$.

Foram ainda encontradas diferenças significativas entre os participantes que tocam instrumento de percussão e aqueles que não o fazem ao nível do compromisso com a música, $(U=1735.50, p<, 001)$. Os participantes que tocam instrumento de percussão $(M=3.39 ; D P=$ .79) apresentam níveis mais elevados nesta dimensão face aqueles que não o fazem $(M=2.60$; $D P=.85)$. Os resultados apontam também para diferenças significativas na dimensão aptidão musical inovadora $(U=907.50, p<, 001)$, sendo os participantes que tocam instrumento de percussão $(M=3.35 ; D P=1.02)$ que pontuam níveis mais elevados face a aqueles que não o fazem $(M=1.83 ; D P=.87)$. Ainda em relação a tocar ou não instrumento de percussão, os resultados apontam diferenças significativas na dimensão efeito psicoativo positivo, $(U=2193.50, p<, 001)$. Assim, são os participantes que tocam instrumento de percussão $(M=4.20 ; D P=.77)$ que reportam/percecionam níveis mais elevados nesta dimensão. No que concerne à aptidão musical inovadora os participantes que ouvem música com fins didáticos $(M=3.36 ; D P=1.10)$ reportam/percecionam níveis mais elevados nesta dimensão face aqueles que não o fazem $(M=$ 1.80; $D P=.82)$. Os resultados apontam para diferenças significativas $(U=1109.00 ; p<, 001)$. 
Existem igualmente diferenças significativas ao nível efeito psicoativo positivo $U=2093.50, \mathrm{p}<$ ,001. Os participantes que ouvem música com fins didáticos $(M=4.31 ; D P=.68)$ apresentam níveis mais elevados nesta dimensão face aqueles que não o fazem $(M=3.66 ; D P=.84)$

Procurou-se ainda analisar as diferenças entre aqueles participantes que ouvem música como objetivo principal e aqueles que não o fazem. Estes resultados são apresentados na tabela 7. Constataram-se diferenças significativas entre os participantes que ouvem música como objetivo principal e aqueles que não o fazem ao nível do Compromisso com a Música, $(U=$ 5353.00, $p<$,o01). Verifica-se um maior compromisso com a música nos os participantes que ouvem música como objetivo principal $(M=2.98 ; D P=(.91)$ e aqueles que não o fazem $(M=2.46$; $D P=.78)$. Há diferenças significativas entre os participantes que ouvem música como objetivo principal e aqueles que não o fazem ao nível da aptidão musical inovadora, ( $U=5769.00, p<$ ,o01). Verifica-se um maior compromisso com a música nos os participantes que ouvem música como objetivo principal $(M=2.30 ; D P=1.10$. $)$ face aqueles que não o fazem $(M=1.79 \mathrm{~S} ; D P=$ .89).

\begin{tabular}{|c|c|c|c|c|c|}
\hline \multicolumn{6}{|c|}{$\begin{array}{l}\text { Tabela } 7 \\
\text { Apresentação dos Resultados Relativos ao Teste de Mann-Whitney utilizando Médias e Desvios-padrão } \\
\text { como Medidas Descritivas (tipo de contexto e motivação: objetivo principal) }\end{array}$} \\
\hline & \multirow{2}{*}{\multicolumn{2}{|c|}{$\begin{array}{l}\text { OUVE MÚSICA COMO } \\
\text { OBJETIVO PRINCIPAL } \\
(n=117)\end{array}$}} & \multirow{2}{*}{\multicolumn{2}{|c|}{$\begin{array}{c}\text { NÃO OUVE MÚSICA COMO } \\
\text { OBJETIVO PRINCIPAL } \\
(n=138)\end{array}$}} & \multirow[b]{3}{*}{$U$} \\
\hline & & & & & \\
\hline & Média & (DP) & Média & $(\mathrm{DP})$ & \\
\hline \multicolumn{6}{|l|}{ BMEQ } \\
\hline Compromisso com a Música & 2.98 & $(.91)$ & 2,46 & $(, 78)$ & $5353.00^{* * *}$ \\
\hline Aptidão Musical Inovadora & 2.30 & $(1.10)$ & 1,79 & $(, 89)$ & $5769.00^{* * *}$ \\
\hline Conexão Social & 3.35 & $(.86)$ & 3,27 & $(, 88)$ & $7684.00^{* * *}$ \\
\hline Reação Emocional & 4.48 & $(.33)$ & 4,38 & $(, 44)$ & $7257,00^{* * *}$ \\
\hline Efeito Psicoativo Positivo & 3.90 & $(.81)$ & 3,63 & $(, 87)$ & $6618,00^{* * *}$ \\
\hline Reação Comportamental à Música & 4.06 & $(.68)$ & $\begin{array}{l}3,78 \\
3,78\end{array}$ & $(, 78)$ & $6379,00^{* * *}$ \\
\hline WEMWBS & 52.90 & $(8.40)$ & 50,89 & $(9,26)$ & $7133.00^{* * *}$ \\
\hline Bem-estar emocional & 13.94 & $(2.70)$ & 13,63 & $(3,15)$ & $7027.50^{* * *}$ \\
\hline Bem-estar social & 18.81 & $(4.72)$ & 18,28 & $(5,09)$ & $7852.50^{* * *}$ \\
\hline Bem-estar psicológico & 28.55 & $(4.86)$ & 28,28 & $(5,30)$ & $7408.00^{* * *}$ \\
\hline Depressão & 3.79 & $(4.44)$ & 4,70 & $(4,80)$ & $7866.50^{* * *}$ \\
\hline Ansiedade & 3.71 & (4.03) & 3,55 & $(3,98)$ & $7862.50^{* * *}$ \\
\hline Stress & 7.19 & $(5.05)$ & 7,99 & $(5,20)$ & $8025,50^{* * *}$ \\
\hline
\end{tabular}

Por último da análise estatística que não se verificaram diferenças significativas entre participantes no que concerne a diferentes estilos de música que ouvem.

\section{Discussão e conclusão}

O estudo desenvolvido teve como objetivos principais verificar a relação que a amostra possui com experiências musicais diversas relacionando essas experiências aos níveis de saúde mental (em termos de bem-estar e psicopatologias). Os principais resultados sugerem que os participantes apresentam níveis relativamente elevados de bem-estar e níveis baixos de psicopatologia (depressão, ansiedade e stress). Pretendeu-se ainda analisar a relação entre as dimensões da experiência da música e a saúde mental. Aqui, globalmente os dados indicaram uma relação positiva entre o grau de compromisso com a música e o nível de bem-estar. Sendo 
que essas afirmações estão em consonância com os resultados de um estudo que sugere que fortes experiências com música tinham o potencial para aumentar o bem-estar de uma amostra de jovens músicos, bem como de ouvintes de música jovem (LAMONT, 2011). Constatou-se também a existência de relação positiva entre o efeito psicoativo positivo e a reação comportamental à música e o nível de bem-estar psicológico. Estes dados ressoam com o estudo levantado por Gabrielsson (2011), no qual os participantes mencionam que foram inspirados a usar música para influenciar o seu próprio humor ou como autoterapia/ autoajuda (self-therapy). Alguma investigação indica que o envolvimento com música individualmente pode melhorar a saúde física e bem-estar emocional (KHALIFA et al., 2003 apud LAMONT, 2011), havendo também outras investigações que têm mostrado que ouvir música na companhia de outros está também associado a fortes experiências positivas (LAMONT, 2011). Outro autor (CROOM, 2015), considera que a participação e a prática de música podem ser produtivamente utilizadas no quotidiano com a finalidade de promover o bem-estar psicológico ou saúde mental. Se acrescentarmos a associação positiva verificada entre o grau de compromisso com a música e o nível de bem-estar pode concluir-se, tal como Lamont (2012) que fazer música faculta uma rota "eudaimônica" para o bem-estar (por meio de compromisso e significado).

Paralelamente, verificaram-se correlações que reportam dimensões menos positivas com relação a saúde mental. Nomeadamente, existe uma correlação positiva entre o grau aptidão musical inovadora e o nível de ansiedade. Considerando os elementos negativos da performance musical, um grande número de estudos tem explorado a ansiedade de desempenho associado à música (KENNY, 2004; WILSON, 2002 apud CUNHA, 2013).

Ansiedade de desempenho afeta cerca de um quarto dos artistas profissionais em grau significativo (Steptoe, 2001 apud LAMONT, 2012) e é comumente definida pelas sensações físicas e mentais experimentadas em situação de performance (LEHMANN et al., 2007 apud LAMONT, 2012). De acordo com Kenny (2003, p. 760 apud CUNHA, 2013), a performance musical requer um alto nível de proficiência em uma extensa área de habilidades e que obter proeminência musical requer execução próxima à perfeição exigindo anos de treino e intensa auto-avaliação. Estes aspetos estão presentes na exigente tarefa de que o músico se defronta no processo de preparação e apresentação de uma obra musical. A conjugação de todos esses elementos, dependendo da pessoa, da tarefa e/ou da situação a ser enfrentada pode resultar em stress físico e psicológico, podendo afetar músicos de diferentes níveis de proficiência (profissionais, estudantes e amadores) (CUNHA, 2013).

No presente estudo verificou-se ainda que o nível de conexão social está associado a maiores níveis de depressão e de ansiedade. Trata-se de uma escala que pode apontar para uma dimensão menos positiva da experiência da música. Segundo LAMONT (2011), fortes experiências com música fornecem ao ouvinte "memórias valiosas e extremamente positivas às quais podem recorrer para gerar níveis mais altos de felicidade" e de acordo com Nelson e FIVUSH (2004), "as memórias autobiográficas relacionam-se com a recordação de eventos passados que foram experienciados pessoalmente”. Verifica-se igualmente uma associação positiva entre o efeito psicoativo positivo e níveis de ansiedade e de stress. A revisão de literatura 
levada a cabo por Miranda et al. (2012), sugere que comportamentos adaptativos e maladaptativos podem coexistir ao escutar música - dependendo da sua força relativa ou frequência -podem levar a mais ou menos internalização psicopatológica, respetivamente. De facto a música pode influenciar as emoções humanas (JUSLIN; SLOBODA, 2012) estando por vezes associada a alguma desregulação de humor nas situações em que o indivíduo desenvolve tendências mais internalizadores das suas emoções (CAMPBELL-SILLS; BARLOW, 2001 apud MIRANDA et al., 2012). Estes dados parecem reforçar a necessidade de continuar a desenvolver estudos que permitam compreender e explicar em maior detalhe e profundidade as relações que parecem existir entre o ouvir música diariamente e sintomas de psicopatologia.

Os resultados apontam ainda para a existência de diferenças significativas entre os participantes que têm outra relação com a música (seja performer, compositor ou professor) face aos que são apenas ouvintes, apresentando os que têm outra relação com a música valores mais elevados nas dimensões compromisso com a música, aptidão musical inovadora e efeito psicoativo positivo. Em todas as formas de exposição musical e interação, dois fatores parecem especialmente importantes explicando as maneiras intensas e profundamente pessoais na qual música formas de vidas dos artistas: curiosidade intelectual e envolvimento emocional. Quando estes dois fatores se misturam, vemos exemplos poderosos de desempenho musical (JUSLIN; SLOBODA, 2012).

Constatou-se que há diferenças significativas entre os participantes que ouvem música com fins didáticos e aqueles que não o fazem ao nível do compromisso com a música, com valores mais elevados nos primeiros. Ouvir música tende a acompanhar ou ser acompanhada por outras atividades do quotidiano, seja a música ou não o principal foco de atenção (LAMONT et al., 2016), neste sentido também é ao nível do compromisso com a música que se verificam diferenças significativas entre os participantes que ouvem música como objetivo principal e aqueles que não o fazem.

Por último, destacamos a inexistência de diferenças entre o Estilo de Música e todas as dimensões avaliadas da experiência com a música, bem-estar e psicopatologia. São diversas as variáveis que influenciam preferências musicais e incluem entre outras, o sexo, a idade e a formação musical. De um estudo, Abeles e Chung (1996 apud HODGES; SEBALD, 2011) concluem que existem poucas ou nenhumas diferenças entre sexo, no que concerne a preferências musicais.

O presente estudo foi guiado pela premissa de que a experiência com a música acontece na relação ativa e complexa entre indivíduo, música e contexto e que é tida como sendo uma das mais prazerosas e satisfatórias atividades do dia a dia (MIRANDA; GAUDREAU, 2011; MIRANDA et al., 2012), e que a experiência da música pode resultar em benefícios para a saúde e bem-estar.(WEINBERG; JOSEPH, 2016).

A música pode ser definida como sendo uma invariante do comportamento humano. $\mathrm{O}$ termo invariante segundo Dubos (1981 apud HODGES; SEBALD, 2011) aplica-se a aspetos 
particulares do comportamento humano que são universais, mas cujas realizações reais diferem amplamente de grupo para grupo. Invariantes decorrem da unidade biológica e psicológica da humanidade. Todos os indivíduos têm as mesmas necessidades, no entanto as expressões dessas necessidades são determinadas culturalmente. A música pode confortar quando se está triste, sublimar os tempos mais felizes, e dar um sentido de unidade; a música é usada para modificar o humor, ampliar sentimentos atuais, libertar a tensão. A música parece ser uma das atividades fundamentais da humanidade (STORR, 1992), e muitas vezes ouvimos música sem sentir qualquer emoção (JUSLIN et al., 2008) apesar de a música ser frequentemente associada a sentimentos de segurança e proximidade com o núcleo familiar os pais e outros parentes próximos (GABRIELSSON, 2011).

Dada a natureza exploratória deste estudo e para uma melhor compreensão dos resultados constata-se a falta de outras variáveis que poderiam contribuir para uma melhor compreensão dos resultados e contrastar com outros estudos existentes. Por exemplo, a introdução de variáveis que medissem e avaliassem o quanto o sujeito participava na música (por exemplo: em média durante quanto tempo por dia estava exposto à música; ou que afunilassem os resultados, por exemplo em vez alternativa à questão da multiplicidade de estilos que ouve, se teria algum estilo de música de eleição e se sim qual). Refere-se ainda que, apesar dos esforços para obter uma grande amostra de participantes, de diversas origens culturais, sociais e musicais, a maioria da amostra é do sexo feminino e sem outra relação com a música. Não se verificar homogeneidade da amostra limita a generalização dos resultados obtidos assim como as fracas correlações encontradas.

Dada a crescente presença da música na nossa vida quotidiana, a investigação de questões relacionadas com e posterior disseminação de conhecimento sobre música e emoção está a tornarse cada vez mais relevante (LUCK, 2014). Alguns autores (HALLAN et al., 2016) apontam para a necessidade de explorar as relações entre a extensão, qualidade e antecedentes de envolvimento individual com a música e a sua identificação com gêneros específicos, tendo em conta as experiências de indivíduos para quem a música é relativamente sem importância. Compreender porque certos indivíduos não se relacionam com a música pode clarificar temas como a habilidade musical, preferência musical, e motivações. A experiência com a música é um fenómeno complexo. Convoca o indivíduo a experienciá-la ora como ilha, ora como ponte para o encontro com o outro, ou como comunhão com algo que o transcende. E ainda assim a música impregnase na nossa vida. (TER BOGT et al., 2010). 


\section{Referências Bibliográficas}

ALMEIDA, Leandro S.; FREIRE, Teresa. Metodologia da investigação em psicologia e educação. Braga: Psiquilíbrios, 2008.

BRADT, J. et al. Music interventions for improving psychological and physical outcomes in cancer patients. Cochrane Database of Systematic Reviews, n. 8, 2011.

CHIN, T. C.; RICKARD, N. S. Beyond positive and negative trait affect: Flourishing through music engagement. Psychology of Well-Being, v. 4, n. 1, p. 25, 2014. ISSN 2211-1522. Disponível em: < http://www.psywb.com/content/4/1/25 >.

CROOM, Adam M. Music practice and participation for psychological well-being: A review of how music influences positive emotion, engagement, relationships, meaning, and accomplishment. Musicae Scientiae, v. 19, n. 1, pp. 44-64, March 1, 2015.

CUNHA, Andre Sinico da. Ansiedade na performance musical: causas, sintomas e estratégias de estudantes de flauta. Dissertação (Mestre em Música). Porto Alegre: Instituto de Artes. Programa de PósGraduação em Música Universidade Federal do Rio Grande do Sul, 2013.

GABRIELSSON, Alf. Strong experiences with music: music is much more than music. New York: Oxford University Press, 2011.

GROARKE, J. M.; HOGAN, M. J. Enhancing wellbeing: An emerging model of the adaptive functions of music listening. Psychology of Music, 2015.

HALLAN, Susan; CROSS, Ian; THAUT, Michael. Where now? In: HALLAN, S.; CROSS, I., et al (Ed.). The Oxford Handbook of Music Psychology. 2. Oxford: Oxford University Press, 2016. cap. 55, pp. 905-913.

HENDERSON, Luke; KNIGHT, Tess. Integrating the hedonic and eudaimonic perspectives to more comprehensively understand wellbeing and pathways to wellbeing. International Journal of Wellbeing, v. 2, n. 3, pp. 196-221, 2012. ISSN 11798602.

HODGES, Donald A.; SEBALD, David C. Music in the human experience: an introduction to music psychology. New York: Routledge, 2011.

JUSLIN, Patrik N. et al. An experience sampling study of emotional reactions to music: listener, music, and situation. Emotion, v. 8, n. 5, pp. 668-83, Oct 2008.

JUSLIN, Patrik N.; SLOBODA, John A. Handbook of music and emotion: Theory, research, applications. Oxford: Oxford University Press, 2012.

KEYES, C. L. M. The mental health continuum: from languishing to flourishing in life. Journal of Health and Social Behavior, v. 43, n. June, pp. 207-222, 2002.

Mental illness and/or mental health? investigating axioms of the complete state model of health. Journal of Consulting and Clinical Psychology, v. 73, pp. 539-548, 2005.

LAMERS, Sanne M. A. et al. Evaluating the psychometric properties of the mental health Continuum-Short Form (MHC-SF). Journal of Clinical Psychology, v. 67, n. 1, pp. 99-110, 2011. ISSN 1097-4679.

LAMONT, Alexandra. University students' strong experiences of music: Pleasure, engagement, and meaning. Musicae Scientiae, v. 15, pp. 229-249, 2011.

Emotion, engagement and meaning in strong experiences of music performance. Psychology of Music, v. 40, n. 5, pp. 574-594, 2012. ISSN 0305-73561741-3087.

LAMONT, Alexandra; GREASLEY, Alinka; SLOBODA, John. Choosing to hear music: motivation, process, and effect. In: HALLAN, S.; CROSS, I., et al (Ed.). The Oxford handbook of Music Psychology. 2. Oxford: Oxford University Press, 2016. cap. 43, pp. 711-724.

LEVITIN, Daniel J. Uma paixão humana. O seu cérebro e a música. Lisboa: Eitorial Bizâncio, 2013.

LUCK, G. Music and Emotion: Empirical and Theoretical Perspectives. Musicae Scientiae, v. 18, n. 3, p. 255, 2014. ISSN 1029-8649.

MACDONALD, Raymond; KREUTZ, Gunter; MITCHELL, Laura. What is music, health, and wellbeing and why is it important? In: MACDONALD, R.; KREUTZ, G., et al (Ed.). Oxford: Oxford University Press, 2012. cap. 1, pp. 3-11.

MARATOS, A. et al. Music therapy for depression. Cochrane Database of Systematic Reviews, n. 1, 2008.

MARQUES, Alexandra. A influência da música na saúde mental e bem-estar: um estudo exploratório. Dissertação (Mestrado em Psicologia Clínica e da Saúde). Porto: Faculdade de Ciências Humanas e Sociais, Universidade Fernando Pessoa, 2017. 
MARQUES, Alexandra; FONTE, Carla. Questionário Breve da Experiência da Música Faculdade de Ciências Humanas e Sociais, Universidade Fernando Pessoa, 2017.

MIRANDA, D. et al. Music listening and mental health: variations on internalizing psychopathology. In: MACDONALD, R.; KREUTZ, G., et al (Ed.). Music, health, and wellbeing. Oxford: Oxford University Press, 2012. cap. 34, pp. 513-529.

MIRANDA, D.; GAUDREAU, P. Music listening and emotional well-being in adolescence: A person- and variable-oriented study. Revue Européenne de Psychologie Appliquée/European Review of Applied Psychology, v. 61, n. 1, p. 1-11, 1// 2011. ISSN 1162-9088. Disponível em: < http://www.sciencedirect.com/science/article/pii/S1162908810000605 >.

NELSON, K.; FIVUSH, R. The emergence of autobiographical memory: a social cultural developmental theory. Psychol Rev, v. 111, n. 2, pp. 486-511, Apr 2004. Disponível em: < https://www.ncbi.nlm.nih.gov/pubmed/15065919 >.

NORTH, A. C.; HARGREAVES, D. J. The functions of music in everyday life: Redefining the social in music psycholy. Psychology of Music, v. 27, p. 71-83, 1999.

NORTON, Peter J. Depression Anxiety and Stress Scales (DASS-21): Psychometric analysis across four racial groups. Anxiety, Stress, \& Coping, v. 20, n. 3, pp. 253-265, 2007/09/01 2007. ISSN 1061-5806. Disponível em: < http://dx.doi.org/10.1080/10615800701309279 >.

PAIS-RIBEIRO, J. L.; HONRADO, Ana; LEAL, Isabel. Contribuição para o estudo da adaptação Portuguesa das Escalas de Ansiedade, Depressão e Stress (EADS) de 21 itens de Lovibond e Lovibond. Psicologia, Saúde \& Doenças, v. 5, pp. 229-239, 2004. ISSN 1645-0086. Disponível em: < http://www.scielo.mec.pt/scielo.php?script=sci arttext\&pid=S1645-00862004000200007\&nrm=iso $>$.

PERETZ, Isabelle. Towards a neurobiology of musical emotions. In: JUSLI, P. N. e SLOBODA, J. A. (Ed.). Handbook of music and emotion: Theory, research, applications. Oxford: Oxford University Press, 2012. cap. 5, pp. 99-126.

RICKARD, N. S. Editorial for "Music and Well-Being" special issue of PWB. Psychology of Well-Being, v. 4, n. 1, p. 26, 2014. ISSN 2211-1522. Disponível em: < http://www.psywb.com/content/4/1/26 >.

RYAN, R. M.; DECI, E. L. On happiness and human potential: a review of research on hedonic and eudaimonic well-being. Annual Review of Psychology, v. 52, pp. 141-166, 2001.

SACKS, Oliver. Musicofilia- Histórias sobre a música e o cérebro. Lisboa: Relógio D'Água, 2008.

SELIGMAN, Martin E. P. Felicidade autêntica: os princípios da psicologia positiva. Cascais: Editora Pergaminho SA, 2011.

STORR, Anthony. Music and the mind. New York: Ballantine Books, 1992.

TER BOGT, T. F. M. et al. Moved by music: A typology of music listeners. Psychology of Music, v. 39, n. 2, pp. 147-163, 2010. ISSN 0305-7356

VÄSTFJÄLL, Daniel; JUSLIN, Patrik N.; HARTIG, Terry. Music, subjective wellbeing, and health: the role of everyday emotions. In: MACDONALD, R.; KREUTZ, G., et al (Ed.). Music, health and wellbeing. New York: Oxford University Press Inc., 2012. cap. 12, pp. 405-423.

WEINBERG, M. K.; JOSEPH, D. If you're happy and you know it: Music engagement and subjective wellbeing. Psychology of Music, 2016. ISSN 0305-73561741-3087.

WERNER, Paul D.; SWOPE, Alan J.; HEIDE, Frederick J. The Music Experience Questionnaire: Development and Correlates. The Journal of Psychology, v. 140, n. 4, pp. 329-345, 2006/o7/01 2006. ISSN 0022-3980. Disponível em: < http://dx.doi.org/10.3200/JRLP.140.4.329-345 >.

. Ethnicity, Music Experience, and Depression. Journal of Music Therapy, v. 46, n. 4, pp. 339-358, December 21, 2009 2009. Disponível em: < http://jmt.oxfordjournals.org/content/46/4/339.abstract >.

WESTERHOF, G. J.; KEYES, C. L. M. Mental illness and mental health: the two continua model across the lifespan. Journal of Adult Development, v. 17, pp. 110-119, 2010. Disponível em: < https://www.ncbi.nlm.nih.gov/pmc/articles/PMC2866965/pdf/10804_2009_Article_9082.pdf $>$. 